\title{
To the problem of observation of two-level tunneling states in supercooled liquids, glass-forming polymers, orientational glasses, and metallic glasses via configurational entropy
}

\author{
Valery B. Kokshenev \\ Departamento de Física, Universidade Federal de Minas Gerais, Instituto de Ciências Exatas, \\ Caixa Postal 702, CEP 30123-970, Belo Horizonte, Brazil \\ E-mail: valery@fisica.ufmg.br
}

Received January 29, 2009

\begin{abstract}
From general perspectives, there is no conceptual gap between the structural and orientational glasses. Both kinds of glasses expose universal features during primary relaxation (Low Temp. Phys. 33, 617 (2007)). However, in spite of much efforts made to observe generic two-level systems (TLS) in polymers, organic liquids, and plastic crystals via thermodynamic measurements, no similarity unifying glass formers was established. Re-analyzing a number of experimental studies, it is revealed that no renormalization conditions imposed on occupation numbers of structural units, relaxing to the glass state, were taken into consideration by the experimentalists. In this study, the effective-cluster approach is applied to configurational (excess liquid-over-solid) entropy measured in both supercooled liquids and crystals through the heat capacity. As the result, new relationships between the observable thermodynamic and dynamic characteristics are found for molecular liquids, polymers, and networks on the basis of available from the literature data. Thereby, new constraints of structure relaxation are shown to give strong evidence for the existence of TLS-type embryos of glassy structure in all studied glass formers.
\end{abstract}

PACS: 61.41.+e Polymers, elastomers, and plastics;

61.43.Fs Glasses;

64.70.P- Glass transitions of specific systems.

Keywords: tunneling states, structural glass transformations, supercooled liquids.

\section{Introduction}

Structural glass transformation in supercooled liquids is one of the long-standing fundamental problems of condensed matter physics. A process of vitrification is followed by the formation of intermediate metastable states in which a dramatic increase in viscosity and anomalous temperature behavior of transport characteristics is commonly encountered above the glass transformation temperature $T_{g}$, established by scanning calorimetry.

From the macroscopic point of view, no conceptual gap exists between the supercooled states in liquids and spin- (metallic and nonmetallic) glasses, orientational (dipolar and quadrupolar) glasses, or structural (molecular and polymeric) glasses. As it have been recently demonstrated via a generalized theoretical framework [1], a cooperative process of glass formation can be treated in terms of material-abstract relaxing units, whose relaxation dynamics is driven by late-time large-cluster spatial correlations. It has been shown that the universal (material-independent) features of the primary relaxation under cooling are stipulated by the slow growing of correlations, as well as by self-similarity of the mesoscopic-scale hierarchical structure of these correlations (see also Ref. 2). Though a specification of correlations depends on the chosen theoretical scheme, their structure similarity is well pronounced by the observation of weakly material-dependent parameters. In this study, the effective clusters [1] considered near the ergodic- nonergodic crossover temperature $T_{e}$ [3] are characterized thermodynamically by configurational entropy, attributed to the excess liquid-over-solid entropy observable via heat capacity in both supercooled liquids and crystals. 
Both structural glasses or orientational glasses having no long-range positional or orientational order between nonpolar or polar molecules are characterized by the formation of correlated metastable states. Orientational glasses, belonging to the class of networks within the entire family of glass formers, are divided, in turn, into two groups. One group includes structurally disordered mixed and doped molecular crystals and another one is formed by plastic crystals not affected by vibrations of the regular crystalline lattice. Similarly to supercooled liquids (treated as a pattern of structural glasses), orientational glass in plastic crystals plays the role of ideal model of orientational glass. Below we introduce material-independent description of glass-forming materials and employ the thermodynamic models of supercooled liquids for plastic crystals.

In 1972, to describe universal behavior of amorphous solids, independent from their chemical composition, Anderson with co-workers [4] and Phillips [5] proposed famous tunneling two-level-system (TLS) description. Remarkably, the TLS hypothesis was anticipated by Adam and Gibbs (AG) model [6], who suggested, for metastable states in supercooled liquids, a two-state minimal-size clusters (called by them cooperatively rearranging regions) characterized by the minimal configurational entropy $s_{a}=k_{B} \ln \Psi$, with $\Psi=2$. However, in spite of much efforts made to verify the TLS hypothesis via thermodynamic measurements in glass forming polymers (with $\Psi=3$ found) [7], organic liquids ( $\Psi>>2$ ) [8], and plastic crystals (e.g., $\Psi=6$ for cyanoadamantane) [7] no TLS similarity unifying glasses was established. In this study we show that the experimentalists did not take into consideration the renormalization conditions imposed on occupation numbers of structural units relaxing to the glass state. As the result, new relationships between the observable thermodynamic and dynamic characteristics are provided for molecular liquids, polymers, and networks.

\section{Background}

\section{Phenomenological and model forms}

The phenomenological Vogel-Fulcher-Tammann (VFT) fitting form, namely

$$
\tau_{T}^{(V F T)}=\tau_{\min } \exp \left(\frac{D T_{0}}{T-T_{0}}\right),
$$

which also reads as

$$
\log _{10} \tau_{T}^{(V F T)}=\log _{10} \tau_{\min }^{(V F T)}+\frac{B}{T-T_{0}}
$$

with $B=D T_{0} / \ln 10$, is widely used to describe the nonArrhenius temperature behavior of the structural relaxation times observed in amorphous liquids and solids; $D$ is the so-called strength index [9] and $T_{0}$ is the VFT temperature. Proposed in the $1920 \mathrm{~s}$, Eq. (1) performs well within the temperature range established as $T_{g} \leq T<T_{c}$ [10], where $T_{c}$ is the crossover temperature $T_{c}$, which separates the moderately and strongly supercooled (SCL) states [10], distinguished in the mode coupling theory [11]. The pre-factor $\tau_{\min }^{(\exp )}=10^{-14 \pm 2} \mathrm{~s}$ reflects the Debye molecule vibrational times. For numerical data on the phenomenological parameters of the forms (1) and (2) established for glass-forming liquids, polymers, and orientational glasses, see Tables 1 and 2 in Ref. 10, and Table 1 in Ref. 12, respectively.

In the AG model, the dynamic properties of SCLs are described [6] by

$$
\tau_{T}^{(A G)}=\tau_{\min }^{(A G)} \exp \left[\frac{\Delta \mu^{(A G)} n_{T}}{k_{B} T}\right],
$$

obtained through the average transition probability $1 / \tau_{T}^{(A G)}$ of the smallest-size cooperatively rearranging regions (CRRs). Here $\Delta \mu^{(A G)}$ is the molar (solid-over-liquid excess) chemical potential, approximated by a constant, whereas $n_{T}$ stands for the mean number of molecules which constitute the rearranging region. Equation (3) was deduced from thermodynamic consideration, given by

$$
\log _{10} \tau_{T}^{(A G)}=A+\frac{C}{T \Delta S_{T}^{(A G)}}
$$

with

$$
\frac{C}{\Delta S_{T}^{(A G)}}=\frac{\Delta \mu^{(A G)} n_{T}}{k_{B} \ln 10},
$$

where $\Delta S_{T}^{(A G)}$ is the molar configurational entropy, defined as the excess liquid-over-solid entropy in the SCL molecular system. Adam and Gibbs treated this system as an ensemble of $N_{A} / n_{T}$ of equal noninteracting clusters ( $N_{A}$ is Avogadro's number), which require the minimum configurational entropy $s_{\min }^{(C R R)}\left(=s_{a}\right)$ for the formation of a solid-liquid boundary. The total molar configurational entropy can be therefore estimated through the AG proposal

$$
\Delta S_{T}^{(A G)}=s_{\min }^{(A G)} \frac{N_{A}}{n_{T}}
$$

given in Eq. (20) of Ref. 6. Furthermore,

$$
s_{\min }^{(C R R)}=k_{B} \ln \Psi_{\min }^{(A G)}, \quad \Psi_{\min }^{(A G)}=2
$$

was suggested [6] as a first estimate for the lowest (critical) entropy, where $\Psi_{\min }$ is the number of distinct configurations available for the formation of the smallest cooperatively rearranging region. Equations (3) and (5) provide the widely employed Eq. (4), where the AG model fitting parameters 


$$
A=\log _{10} \tau_{\min }^{(A G)} \text { and } C=\frac{N_{A} s_{\min }^{(C R R)}}{k_{B} \ln 10} \Delta \mu^{(A G)}
$$

are commonly derived from the high-temperature data. In Ref. 13, the SCLs were studied simultaneously on the basis of the dynamical data $\tau_{T}^{(\exp )}$, derived from the dielectric loss spectra, and the thermodynamical experimental data on the configurational entropy, namely

$$
\Delta S_{T}=\int_{T_{K}}^{T} \frac{\Delta C_{T}}{T} d T, \Delta C_{T}=C_{T}^{(\text {liq })}-C_{T}^{(\mathrm{sol})},
$$

evaluated through the excess liquid-over-solid isobaric specific heat $\Delta C_{T}$. The thermodynamic Kauzmann temperature $T_{K}$ is defined by the condition $\Delta S_{K}=0$. Taking into consideration the experimental fact that the high-temperature asymptote is observed as $\Delta C_{T}^{(\exp )} \propto T^{-1}$, the $\mathrm{AG}$ model was specified in both thermodynamic and dynamic aspects. First, the configurational entropy (8) was found [13] in the explicit interpolation form, namely

$$
\Delta S_{T}^{(\text {int })}=\Delta S_{\infty}\left(1-\frac{T_{K}}{T}\right) \text { with } \Delta S_{\infty}=\frac{C}{B} .
$$

Here $B$ is the VFT-form dynamic parameter, defined in Eq. (2), while the thermodynamic parameter $C$ is given in Eq. (4).

Adopting the dynamic-thermodynamic correspondence for the primary timescale, we introduce here the minimum relaxing-unit sizescale $n_{a}$ through the relations

$$
n_{T}=n_{T}^{(V F T)}=n_{T}^{(A G)}=\frac{n_{a}}{1-T_{0} / T}
$$

with

$$
n_{a}=n_{\min }^{(C R R)}=\frac{k_{B} B \ln 10}{\Delta \mu^{(A G)}} .
$$

Here $n_{\min }^{(C R R)}$ stands for the minimum molecular size of the CRRs and $n_{T}^{(A G)}$ specifies it for the AG model, in Eq. (3). Furthermore, our consideration must be completed by the lower limit timescale $\tau_{a}$ and critical entropy $s_{a}$, namely

$$
\tau_{\min }^{(V F T)}=\tau_{\min }^{(A G)}=\tau_{a} \text { and } s_{\min }^{(A G)}=s_{a}
$$

The examination made with help of Eqs. (10) and (11) results in

$$
B=\frac{\Delta \mu_{g} n_{a}^{(S L)}}{k_{B} \ln 10} \text { and } C=\frac{\Delta \mu_{g} N s_{a}}{k_{B} \ln 10},
$$

where additional parameters $\Delta \mu_{g}=\Delta \mu^{(A G)}$ and $n_{a}=n_{a}^{(S L)}$ are introduced for the SL state. For further details, see Eq. (25) in Ref. 10.

\section{How to observe the TLS}

A famous concept on tunneling excitations, attributed to the ground glassy state in amorphous solids [4,5], was anticipated in the AG theory [6]. Indeed, the two-levelsystem (TLS) hypothesis was employed in explicit form in Eqs. (5) and (6), as a lower-limit critical condition in the formation of solid-like CRRs in the normal liquid of $N_{A}$ molecules.

The thermodynamical observation, through the heat capacity data, of the mean occupation number in the solid-like clusters $n_{T}^{(T D)}$ is commonly based on the AG proposal (5) reading as

$$
n_{T}^{(T D)}=\frac{N_{A} s_{a}}{\Delta S_{T}},
$$

with the configurational entropy $\Delta S_{T}$ estimated through Eq. (8). This can exemplified by the data [8] on CRR molecular size at $T_{g}$, such as $n_{g}^{(T D)}=3.8,4.3$, and 4 , observed through Eq. (13) in, respectively, 3-bromopentane, OTP, and toluene in the SL state, as well as by $n_{\text {glass }}^{(T D)}=4.5,4.6$, and 7.5 obtained through the excess entropy $\Delta S_{T}^{\text {(glass) }}$ attributed to the glass state. Apart from the cluster size, Eq. (13) was used for observation of the CRR configurational entropy $s_{a}=k_{B} \ln \Psi_{\min }$ (6), treated as the model-independent fundamental parameter. Being normalized at the melting temperature, Eq. (13) resulted [7] in $\Psi_{\text {min }}^{(C A D)}=6$, for the number of available local-axis equilibrium states in supercooled cyanoadamantane, supported by its crystalline symmetry. Also, $\Psi_{\min }^{(\mathrm{pol})}=3$ and $\Psi_{\min }^{(\text {liq })}>>2$ were correspondingly derived in glass-forming polymers [7] and expected in organic liquids [8,7].

In view of the proposed study, the following comments can be made. As was discussed in Fig. 3 in Ref. 12 and Fig. 6 in Ref. 1, plastic crystals are not dynamically consistent with the structurally disordered glass formers, including SCLs. Also, as independently observed in Ref. 8, plastic crystals, unlike liquids and polymers, do not obey the AG-VFT correspondence, discussed in Eq. (9). Nevertheless, the AG qualitative estimate (13) is not specified by the structure formation of the underlying relaxing units and, therefore, it can be tested in all glass formers. Furthermore, attention should be paid to the normalization conditions. The first CRR appears below the Arrhenius crossover temperature $T_{A}$, and thus $n_{T} \equiv 0$, in ergodic phase $A$ [3] at $T>T_{A}$, which usually lies below $T_{m}$. This means that the high-temperature asymptote is inappropriate for the moderately cooled liquid state, that raises a question concerning the accuracy of the asymptotic AG-VFT relation $\Delta S_{\infty}^{(\exp )}=C / B(9)$, with all consequent relations given in Eqs. (12). However, this remark does not affect the experimentally observed [13] equation $\Delta S_{T}^{(\text {int })}=\Delta S_{T}^{(\exp )}(9)$, which is now specified for 


$$
\Delta S_{T}^{(\exp )} n_{T}=\Delta S_{\infty}^{(\exp )} n_{a}^{(S L)}, \quad T_{g} \leq T<T_{c},
$$

with the help of Eq. (10).

So as evaluate the number of locally equilibrated states in strongly SCLs, associated with $\Psi_{\min }^{(S L)}$, let us determine parameter $\Delta S_{\infty}^{(\exp )}$ (14) through the jump in the excess specific heat $\Delta C_{g}^{(\exp )}$, observed at $T_{g}$ [14]. If one adopts a form $\Delta C_{T}^{(\exp )}=\Delta C_{g}^{(\exp )} T_{g} / T$, compatible with the analysis given in Ref. 13, the useful relation

$$
\Delta S_{\infty}^{(\exp )}=\Delta C_{g}^{(\exp )} \frac{m_{g}}{m_{g}-m_{g}^{*}}
$$

can be deduced from Eqs. (8), (9) and Eq. (22) in Ref. 1. Taking into consideration Eqs. (14), (15) and (10), the AG proposal, given by Eq. (5) and specified in Eq. (6), reads for the ergodic supercooled liquid (SL) state as

$$
\frac{s_{a}^{(S L)}}{k_{B}}=\ln \Psi_{\min }^{(S L)}=\frac{\Delta C_{g}^{(\exp )}}{R} \frac{n_{g} m_{g}^{*}}{m_{g}-m_{g}^{*}} .
$$

Here $R=k_{B} N_{A}$ is gas constant and the cluster molecular size $n_{g}$ is expected to be bounded by $n_{g}^{(\bmod )}=8 \pm 1[10]$. We have therefore shown, that the number of the energy minima on the potential energy landscape can be observed through the specific heat jump data, with the help of Eq. (16).

Also, we offer a qualitative estimate for the absolute minimum of the configurational entropy $s_{a}$ in glass-like clusters, which are presumably emerge below $T_{g}$. This can be defined through the relation

$$
\Delta S_{e}^{\text {(glass) }} n_{b}=N_{A} s_{a} n_{g},
$$

which is an adaptation of the AG proposal to the temperature domain $T_{e} \leq T \leq T_{g}$. The left side of Eq. (17) is given as a lower limit for the low-dense clusters, presented by the excess entropy $\Delta S_{e}^{\text {(glass) }}$, taken at the ergodic transition temperature $T_{e}$ [3] and the right side is approximated by the minimum configurational entropy for CRRs formed at $T_{g}$, approximated by $s_{g}^{(C R R)}=s_{a} n_{g}$. With the help of $n_{T}(10)$ at $T_{g}$, and the ratio $n_{b} / n_{a}^{(S L)}=T_{e} / T_{0}$, deduced from Eq. (5) in Ref. 3, one obtains

$$
\frac{s_{a}^{(\min )}}{k_{B}}=\ln \Psi_{\min }^{(\text {glass })}=\frac{T_{e}}{T_{0}} \frac{m_{g}^{*}}{m_{g}} \frac{\Delta S_{e}^{(\text {glass })}}{R}
$$

following from Eq. (17).

For a rough numerical estimates, one adopts $\Delta S_{e}^{\text {(glass) }} \approx$ $\approx \Delta S_{g}^{(\exp )}$ laying between 20 and $25 \mathrm{~J} / \mathrm{K}$ mole in SCLs [13], whose fragility number is bound by $35 \leq m_{g} \leq 81$. This yields the optimistic domain $1.5<\Psi$ min ${ }_{\text {(glass })}<2.3$, attributed to the nuclei of the glassy structure.

More specific estimates provided in Table 1 follow from the auxiliary relation

$$
\frac{n_{b}}{n_{g}}=\frac{N_{A} s_{a}}{\Delta S_{e}^{\text {(glass })}}=\gamma_{c} \frac{T_{c}-T_{0}}{B}
$$

following from Eq. (6) in Ref. 3. This yields

$$
\frac{s_{a}^{(\min )}}{k_{B}}=\ln \Psi_{\min }^{(\text {glass })}=\gamma_{c} \frac{T_{c}-T_{0}}{B} \frac{\Delta S_{e}^{\text {(glass })}}{R} .
$$

In Table 1 the estimates are provided on the basis of Eq. (19) and dynamic and thermodynamic data available from the literature.

As seen from Table 1, the TLS are well observable in supercooled liquids, instead of generally established [8] $\Psi>>$ 2. Similar to the case of Eq. (13), the estimate, pro-

Table 1. Estimation of the minimum number of local tunneling states $\Psi$ during formation of glassy macroscopic state

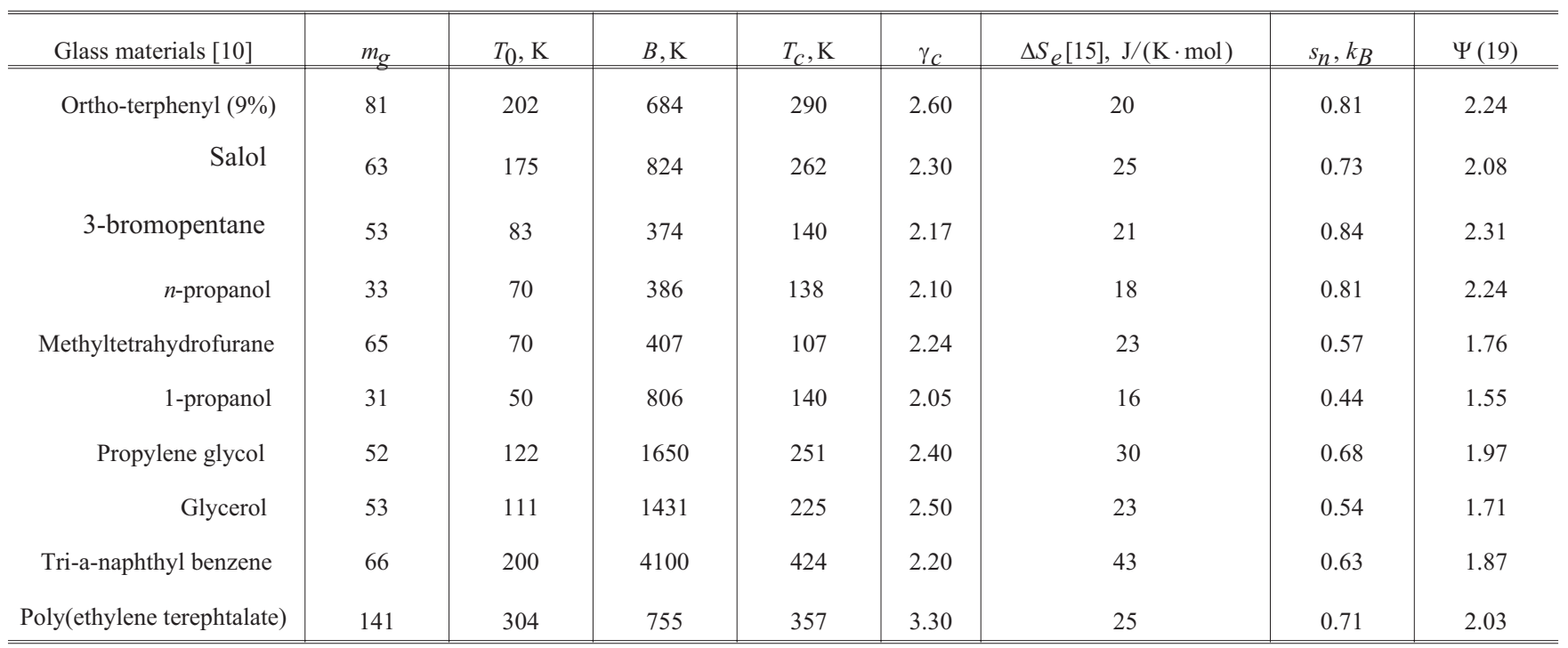


posed in Eq. (18), is not limited by SCLs. It can be applied to amorphous polymers and metallic alloys, with the help of the $T_{e}$ data given, respectively, in Figs. 20 and 21 in Ref. 2. These data challenge new measurements of configurational entropy. In vitreous network solids, the direct observation of TLS was reported in Ref. 16.

\section{Conclusion}

We have shown how the renormalization conditions imposed on occupation numbers of structural units relaxing to the glass state should be taken into consideration for the observation of TLS in glass forming materials. As the result, new relationships between the observable thermodynamic and dynamic characteristics are provided for molecular liquids, polymers, and networks. The estimates made on the basis of available from the literature data give evidence for the observation of TLS-type embryos of glassy structure in all glass formers. More generally, we have shown how the earlier proposed cluster ergodicnonergodic description [3] may provide a new tool for accounting for a number local-equilibrium states in Goldstein's energy landscape.

The financial support by CNPq is acknowledged.

1. V.B. Kokshenev, Fiz. Nizk. Temp. 33, 805 (2007) [Low Temp. Phys. 33, 617 (2007)].

2. V.B. Kokshenev, Heterostructured Molecular Clusters in Supercooled Liquids and Other Glass-Forming Materials:
Dynamic and Thermodynamic Appearance in the Primary Structural Relaxation, Chapter in Atomic and Molecular Cluster Research, Y.L. Ping (ed.), Nova Science Publishers, New York (2006).

3. V.B. Kokshenev, Solid State Commun. 119, 429 (2001).

4. P.W. Anderson, B.I. Halperin, and C.M. Varma, Philos. Mag. 25, 1 (1972).

5. W.A. Phillips, J. Low Temp. Phys. 7, 351 (1972).

6. J.H. Gibbs and G. Adam, J. Chem. Phys. 43, 139 (1965).

7. S. Takahara, O. Yamamuro, and T. Matsuo, J. Phys. Chem. 99, 9589 (1995).

8. O. Yamamuro, M. Ishikawa, I. Tsukushi, and T. Matsuo, in: Slow Dynamics in Complex Systems, M. Tokuama and I. Oppenheim (eds.), Eighth Tohwa University International Symposium, AIP (1999), p. 513.

9. R. Böhmer, K.L. Ngai, C.A. Angell, and D.J. Plazek, J. Chem. Phys. 99, 4201 (1993).

10. V.B. Kokshenev, P.D. Borges, and N.S. Sullivan, J. Chem. Phys. 122, 114510 (2005).

11. W. Götze and L. Sjögen, Rep. Prog. Phys. 55, 241 (1992).

12. V.B. Kokshenev and N.S. Sullivan, Phys. Lett. A208, 97 (2001).

13. R. Richert and C.A. Angell, J. Chem. Phys. 108, 9016 (1998).

14. O. Yamamuro, I. Tsukushi, T. Matsuo, K. Takeda, T. Kanya, and K. Kaji, Progr. Theor. Phys. 126, Suppl., 93 (1997).

15. J.H. Magill, J. Chem. Phys. 47, 2802 (1967).

16. F. Ladieu, J. Le Cochec, P. Pari, P. Trouslard, and P. Ailloud, Phys. Rev. Lett. 90, 205501 (2003). 